\title{
DISTRIBUTED SIMULATION IN MANUFACTURING USING HIGH LEVEL ARCHITECTURE
}

\author{
J. Rodríguez Alvarado*, R. Vélez Osuna ${ }^{* *}$, R. Tuokko* \\ *Tampere University of Technology, Institute of Production Engineering, \\ Automatic Manufacturing and Assembly Laboratory, P.O. BOX 589, \\ FIN-33101 Tampere, Finland. \\ Tel +358331154487, Fax +358331152753 \\ jose.rodriguezalvarado@tut.fi, and reijo.tuokko@tut.fi.
}

**Visual Components Oy, Korppaanmäentie 17 CL6, 00300 Helsinki, Finland, Tel +35893232250

ricardo.velez@visualcomponents.com

\begin{abstract}
Distributed simulation has the potential to become widely applicable for geographically-dispersed manufacturing environments, as is the case with desktop manufacturing or rapidly deployable micro-assembly stations. The work presented here discusses in detail the theory of the distributed manufacturing simulation infrastructure based on the IEEE HLA standard and clarifies with practical examples the potential of this approach in modeling and simulation of assembly systems in complex and distributed manufacturing environments.
\end{abstract}

Keywords distributed simulation in manufacturing, HLA

\section{Introduction}

Manufacturing is a critical industry for all major economies. Every individual and industry depends on manufactured goods which makes manufacturing crucial to the national economies. Competition is increasingly hard and globalisation is leading to worldwide distribution of production, products and services, affecting all countries and economical regions. At the same time markets are changing. Customers call for faster product changes and demand products which are increasingly targeted to individual needs. Mass production is therefore replaced by customised and personalised production of individual products.

Manufacturing companies are now living in a time where fast prototyping is the rule and products should be assembled fast and accurately. Moreover, the life cycle of products is decreasing constantly on the market and costs have to be cut everywhere. One way to keep up with all those changes has been shrinking of the value 
chain with the main objective of producing high quality and fast deployable customised products.

Constructing a prototype may be costly, unfeasible, and/or dangerous [1]. Using 3-D models, designers can study and refine assembly sequences for ease of execution, and identify problems that otherwise might not be detected until significant resources were already committed to production [2].

Consequently, 3-D software had become a fundamental part of manufacturing when designing and simulating virtual environments. 3-D models are not only a visual representation of the real components but also can be a portrayal of performance.

\section{Distributed Simulation}

One of the problems that realistic simulation usually faces is the need of large resources for execution of simulation models with increased complexity. A normal solution to the simulation model complexity problem is to decrease the level of details. This solution allows home-office machines to perform complicated simulations without any radical slowdown in the machine performance.

Distributed simulation has been widely used in numerical simulations and made its path for fifteen years in the virtual environments for military applications. The advantage of using distributed simulations is that each calculation can be done in a single ordinary computer and when the partial result is ready, it is sent to the computer that uses those results for its own calculations, improving the speed and power of the computations. Another advantage is redundancy; in case of a failure of one machine, other computers can handle over the data that the failing computer should have processed.

In the middle of the past decade the US Department of Defense (DoD) came up with the need to standardise the way simulators and simulations could communicate between each other. The result of this standardisation was the High Level Architecture (HLA), with a mandate that simulations still in use and all future developed simulations should be compliant with the HLA standard [3]. HLA was a successful answer to the need of interaction between simulations and simulators from different manufacturers, which later on was defined as Standard 1516 by the IEEE for the open community.

The HLA environment includes certain definitions and components. The main ones are the RTI, the federation object model, the federation and the federate. The Runtime Infrastructure (RTI) is the core of the HLA where many federations can reside. A federation is a named set of federate application and a common Federation Object Data that are used as a whole to achieve some specific objective. A federate is an application that may be coupled with other software applications under a Federation Object Model Document Data and a RTI [4]. A federate can be a simulation or a human interaction like a pilot in a cockpit simulator.

Manufacturing simulations, including those of microfactories, have been until now centralised simulations. This has made it imperative to use powerful computers for extensive or high-detailed simulations. 
Utilising a distributed approach, every part of the simulation can be displayed in high detail, running the simulations on ordinary computers.

\section{The MS2Value project}

The MS2Value project (Modeling and Simulation of Manufacturing Systems for Value Networks) aims at developing a distributed simulation environment for manufacturing operations, building it on top of open source or commercially available components [5]. By using this approach, manufacturing operations could be simulated across the world, where each federate would be running a small, but rich in detail, portion of the simulation in each machine and all the statistics of the simulation can be retrieved via the RTI. These simulations can run in different geographical locations as shown in Figure 1.

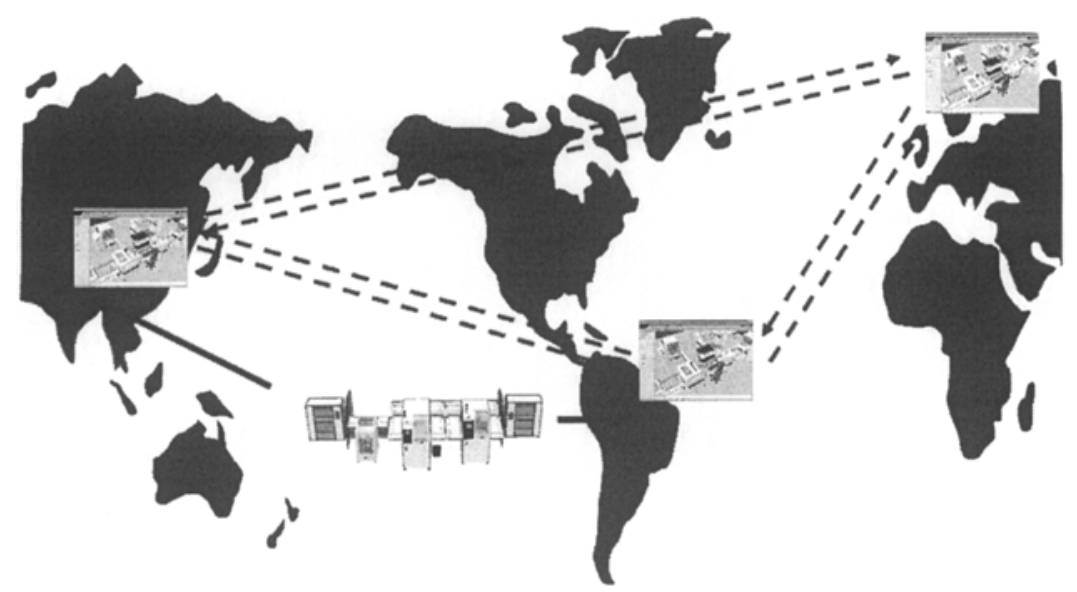

Fig. 1 . Distributed and real time simulation is possible using HLA and SOA

In the MS2Value project, the DS-HLA (Distributed Simulation based on High Level Architecture) application was created to interconnect Visual Components' 3DCreate [6] simulations through an open source RTI (jaRTI) [7]. In each simulation, it is possible to implement one or more federates. These federates would be handling only a part of the microfactory operations and after the product has been processed, the federate would give an update to the federation related with that operation.

Figure 2 shows the architecture developed for the MS2Value project. As is shown, different federates can connect to the RTI via sockets or web services and share information between the 3DCreate applications. It is a HLA rule that all the interactions must be made through the RTI. It is also possible to monitor the federation through the console and verify the state of the federation. 

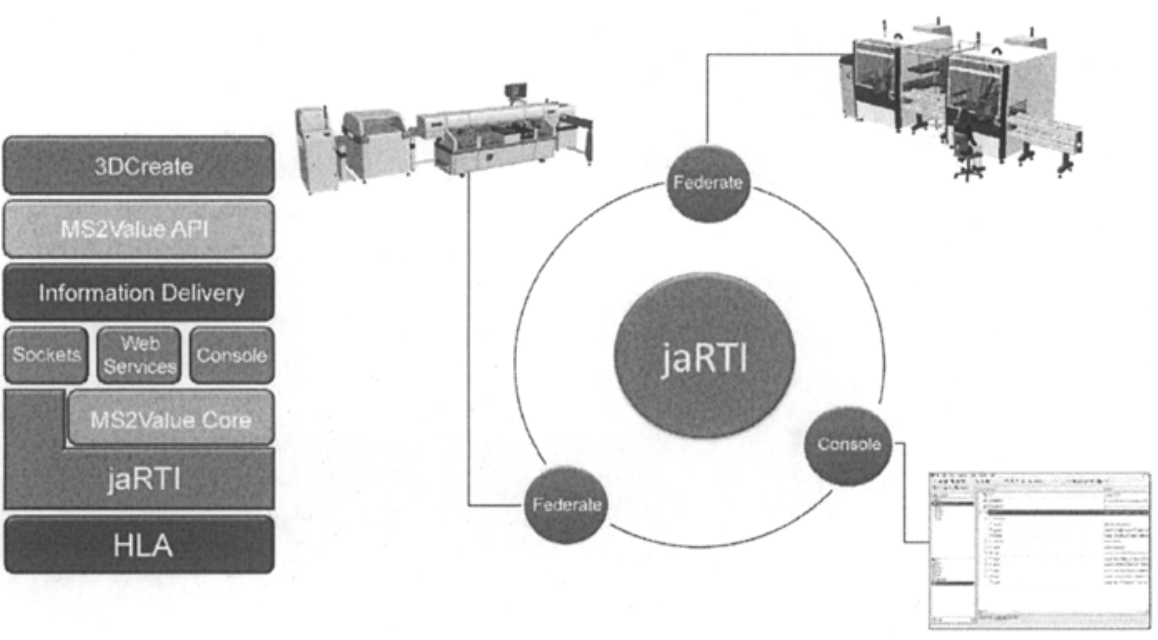

Fig. 2. Architecture of the MS2Value Project

The architecture is designed not only to work with Visual Components' software; any application can be designed to monitor a federate, a parameter or a federation and provide statistical data about the simulation.

\section{Applications of the DS-HLA}

The MS2Value architecture can be used for multiple applications concerning the simulation field. In addition to complex macro-scale assembly systems, simulation of micro and desktop factories can also be challenging, if the models should be accurate in size and details so that the model can give a realistic representation.

By using the DS-HLA application one can simulate the complete process of the micro-assembly station or a micro-factory represented in different machines with high detailed models which would be the main pattern at the time of doing the real implementation. All of these simulations can be done in lightweight processes without overloading the computer's processors.

When modeling an assembly system, some modifications can be done in the process using this approach that would allow comparing an end product going through different processes. For example, a simulation federate assembles an accelerometer and other simulation federate handles the packaging. By connecting more federates to the federation, it would be possible to make a parallel packaging process simulation where we can compare different types of machines, assembly techniques, cycle times, etc.

Figure 4 shows a graphical representation of the example mentioned above. 


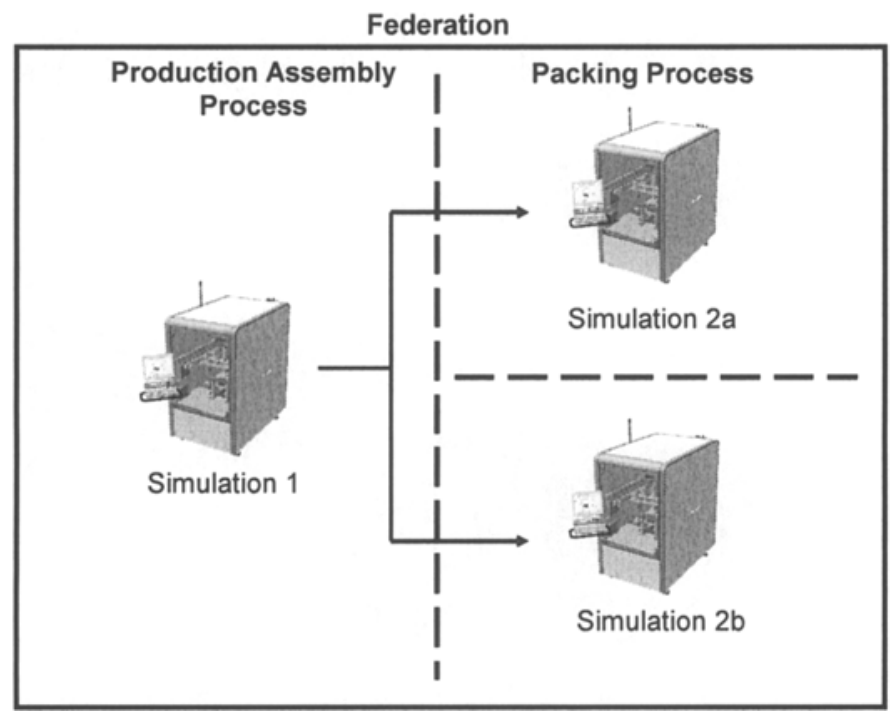

Fig. 4. Diverging of the same product into several processes to weigh their performance

These simulations can grow as the needs of the process designers evolve, models can communicate back and forward and information can be grabbed and stored by other federates in databases or files.

Another theoretical example can be that several micro or desktop factories are simulated in different parts of the globe. Those simulations would offer certain processes that could be used in the production of goods. Using a similar approach to the DS-HLA application, users could design an assembly sequence based on the availability of the services, run the simulation from where it could be possible to monitor the production, get the statistics and validate different scenarios.

\section{Conclusions}

Distributed simulation has the potential to become an important tool for widely distributed manufacturing organisations. Global competition forces more efficient manufacturing and assembly operations which are more and more often distributed over a value network. This kind of networked manufacturing increases the complexity of overall capacity calculations and overall system simulation, and micro and desktop factories are also on this development track. The DS-HLA application developed as part of the MS2Value serves as a theoretical proof of distributed simulation for manufacturing systems. A pilot case study has been developed, but industrial applications are still to be waited for. Development on the DS-HLA will continue still as an open source project, partly funded and developed by Visual Components and research partners, such as Tampere University of Technology. 


\section{References}

1. R. Fujimoto, Parallel and Distributed Simulation Systems, p.4.

2. Integrated Manufacturing Technology Initiative; Integrated Manufacturing Technology Roadmapping Project, Modeling and Simulation (2000) p.3-6.

3. IEEE Std 1516-2000, IEEE Standard for Modeling and Simulation (M\&S) High Level Architecture (HLA) Framework and Rules.

4. Defense Modeling and Simulation Office, United States Department of Defense, High Level Architecture. (August 20, 2007); https://www.dmso.mil/

https://www.dmso.mil/public/transition/hla/

5. R.Velez Osuna, R. Tuokko, Modeling and Simulation as a Tool for Decision Making in Adaptive Value Networks, (International Conference of Concurrent Enterprising ICE, 2006)

6. Visual Components Oy, http://www.visualcomponents.com

7. jaRTI (now renamed poRTIco), http://www.porticoproject.org 